\title{
Laboratory Simulation of Volcano Seismicity
}

\author{
Philip M Benson ${ }^{1} 2^{\star}$, Sergio Vinciguerra ${ }^{3}$, Philip G Meredith ${ }^{1}$ and R Paul Young ${ }^{2}$ \\ *Corresponding author: p.benson@ucl.ac.uk
}

\begin{abstract}
${ }^{1}$ Rock and Ice Physics Laboratory, Department of Earth Sciences, University College London, Gower Street, London, WC1E 6BT, U.K.

${ }^{2}$ Lassonde Institute, University of Toronto, 170 College Street, Toronto, Ontario, M5S 3E3, CANADA.

${ }^{3}$ High Pressure High Temperature Laboratory of Experimental Volcanology and Geophysics, Department of Seismology and Tectonophysics, Istituto Nazionale di Geofisica e Vulcanologia, Via di Vigna Murata 605, 00143 Rome, ITALY
\end{abstract}

Physical processes generating seismicity within volcanic edifices are highly complex and remain not fully understood. We report results from a laboratory experiment in which basalt from Mount Etna volcano (Italy) was deformed and fractured. The experiment was monitored using an array of transducers around the sample to permit full-waveform capture, location and analysis of microseismic events. Rapid, post-failure decompression of the water-filled pore volume and damage zone triggered many low frequency events, analogous to volcanic long period seismicity. The low-frequencies were associated with pore fluid decompression and were located in the damage zone in the fractured sample; these events exhibited a weak component of shear (double-couple) slip, consistent with fluid-driven events occurring beneath active volcanoes. 
Seismicity in volcanoes is intimately related to ground deformation, faulting, and the movement of volcanic fluids within the edifice. The discrimination and interpretation of different types of seismic signals is a central goal for hazard evaluation and mitigation [e.g. $(1,2,3)]$. Volcano-Tectonic (VT) seismicity occurs as the Earth's crust is deformed and fractured by magma movement, resulting in shallow focus earthquakes beneath the volcanic edifice; which frequently provide the first sign of growing unrest [e.g. $(4,5,6)]$. Faulting provides conduits between the deep reservoir and the surface ( 7 , and the final approach to eruption is commonly preceded by accelerating rates in the occurrence of low magnitude VT earthquakes (5). A second type of seismic activity is related to fluid movement, although the details remain uncertain. Low Frequency (LF) events - also known as long period, (LP) and very long period (VLP) events - are observed on all types of active volcanoes, often in swarms preceding eruption. These LF events differ from VT events in frequency range and harmonic signature $(1,2,4)$, and are postulated to be generated from fluid flow and resonance in fractures and conduits within the edifice. When LF signals become continuous or quasi-continuous, volcanic tremor is generated. It has been suggested that LF earthquakes could provide the basis for a method of eruption forecasting (8). However, as other tectonic processes often yield seismic signatures resembling low frequency tremor, for example episodic tremor and slip in subducting regions $(9,10)$, further work is urgently needed on addressing the physical processes responsible for LF event generation in volcanic settings.

Here, we report results from one of a series of laboratory deformation experiments on Etna basalt samples (11), in which we have generated microseismic signals, better known as acoustic emissions (AEs) (12), that exhibit near identical features to those observed during volcanic activity when scaled to seismic frequencies. We deformed cylindrical samples of Etna basalt ( $50 \mathrm{~mm}$ in diameter by $125 \mathrm{~mm}$ long, with a centrally pre-drilled $3 \mathrm{~mm}$ diameter conduit) at an effective confining pressure of $60 \mathrm{MPa}$; representative of pressure conditions approximately 2 $\mathrm{km}$ beneath volcanic edifices $(13,14)$; The initial pore fluid pressure was $20 \mathrm{MPa}$, giving an 
effective confining pressure of $40 \mathrm{MPa}$. Experiments were conducted in two stages. In stage 1, samples were deformed at a constant strain rate of $4 \times 10^{-6} \mathrm{~s}^{-1}$ until brittle failure occurred. This resulted in the creation of a localized shear fault and an associated crack damage zone. In stage 2, the fluid stored in the sample (including both pre-existing microcracks and the new fault damage zone) was rapidly decompressed from the top of the sample (15, Fig. S1.), stimulating rapid fluid flow out of the sample via the damage zone and the pre-drilled conduit. The output of acoustic emissions (AEs) was recorded continuously throughout both phases using an array of 16 transducers. In addition to recording waveforms, the array was used to locate individual AE events using a downhill simplex routine and a triaxial velocity model $(11,15)$. We estimate that our $\mathrm{AE}$ locations are accurate to $\pm 1.5 \mathrm{~mm}$ (11). We also computed source characteristics (mechanisms) of the located events via relative amplitude moment tensor analysis $(16,17)$. Not only does our experiment concur with field data in which fluid movement is frequently a key mechanism behind seismic swarms generally (18), in addition to being ubiquitous in volcanic areas where it is often implicated in rapid volume change processes such as fluid motion in conduits associated with magma decompression (8), but we are also able to resolve moment tensors for independent verification of pore volume changes that are a feature of fluid-driven earthquakes.

We observed a low rate of $A E$ output during initial loading, as pre-existing cracks closed. Activity then increased exponentially as differential stress increased and new, dilatant cracks nucleated and propagated $(11,15,17,19)$. Failure occurred by shear faulting at a failure stress of $480 \mathrm{MPa}$, and 2.9\% axial strain (15, Fig. S2). We recorded 1518 events during deformation, and located 762. Of these, most were located within the damage zone of the shear fault, or its conjugate (Fig. 1A). They exhibited dominantly double-couple (DC) source characteristics (Fig. $1 \mathrm{~A})$, entirely as expected for the faulting phase of the experiment. We verified that the presence of the central conduit did not significantly affect the mechanics of deformation and failure by conducting identical experiments without a conduit, and obtaining essentially identical results. 
The rapid decompression of the pressurized pore fluid (over $0.2 \mathrm{~s}$ ), by means of a valve at the top of the apparatus (15, Fig. S1), was accompanied by a swarm of $A E$ events again located near the fault damage zone generated during stage 1 (Fig. 1B, Fig. S3). In contrast with the deformation phase of the experiment, the source characteristic of the decompression-related events exhibited low components of shear, but a high volumetric component (Fig. 1B). We therefore postulate that the rapid flow of the pore fluid through a tortuous fracture damage zone, imposed by the rapid decompression, created conditions conducive for the formation of a swarm of $\mathrm{AE}$ events analogous to LF events recorded at active volcanoes. Source mechanisms involving high levels of volumetric change have been widely reported in volcanic areas $(13,20)$ and in areas of tectonic subduction and fault overpressure $(9,10,18)$; all of which have been linked to fluid movement.

Further similarities between field data and our laboratory data can be seen in the frequency domain (Fig. 2). During the rock deformation stage, high frequency $A E$ signals dominated as the shear fault nucleated and propagated across the sample (Fig. 1A). Brittle processes, from millimeter-scale laboratory microcracking to kilometer-scale earthquake rupture, generate high frequency movement and acceleration. In our experiment, the peak in power occurred at a frequency of approximately $600 \mathrm{kHz}$ (Fig. 2A) during sample deformation and fracture. A characteristic feature of volcano seismic events due to fluid movement is their low frequency signature. This type of LF event has principal power components of $1-2 \mathrm{~Hz}(4,12)$, and most authors postulate that resonance within cracks or volcanic conduit flow is responsible for such seismicity, which often show non-double-couple source characteristics $(10,20,21)$. In our experiments, the decompression of the fluid filled pore space resulted in swarms of $A E$ activity with a power frequency of approximately $18-50 \mathrm{kHz}$ (Fig. 2B). The events have noticeably longer, ringing waveforms as compared with those from deformation events, but with noticeable harmonic overtones; features commonly seen in field seismic data $(1,4)$. Experimental studies 
using open ended tubes of both constant and changing section have revealed similar frequency characteristics $(22,23)$. In addition to these two key end-member waveform types, our experiments yielded a high frequency initial component as well as LF characteristic that had features of both VT and LF event types. We postulate that these events occurred when the fluidfilled rock faulted and fluids moved into the fracture. Such waveforms are commonly known as hybrid events in volcano seismology (4).

To investigate the microstructural origin of located AE events, we analyzed backscattered scanning electron microscopy images of the deformed and decompressed sample. As shown in Fig. 3, a complex damage zone formed in the lower half of the sample, dominated by two major, conjugate faults. During decompression, events were located within this damage zone. The detailed locations of two particular AE clusters imply that the fluids producing these events were following highly tortuous pathways, with many pinch-outs and undulating features. Such geometries have long been postulated to be responsible for tremor-type events (24). Field emission SEM observations showed that many of the cracks were filled with broken and comminuted rock (Fig. 3, inset B), as also reported from field observations of fractured magma and obsidian (25). Taken together, these observations suggest that LF events in volcanic areas are generated when hydrothermal fluids (water, steam, dusty gasses and/or magma itself) move through pre-existing crack networks $(4,13)$ comprising both larger faults and their associated fracture damage zones (Fig. 3).

Using a simple size-frequency scaling relationship $(13,26)$, we show that LF events with laboratory length $\left(\mathrm{d}_{\mathrm{L}}\right)$ and frequency $\left(\mathrm{f}_{\mathrm{L}}\right)$ scales $(50 \mathrm{~mm}$ and $18-50 \mathrm{kHz}$, respectively) can be scaled appropriately to data from source dimensions $(\mathrm{dv})$ and seismic frequencies (fv) typical of natural volcanic events (200 m $-1 \mathrm{~km}$ and 1-2 Hz, respectively) (6). AE obeys power law relationships, just as field scale seismicity does, permitting similar statistics to be employed (27, 28). Following the treatment of (13) and (26), one may write $d_{L} \times f_{L}=d_{v} \times f_{v}$, if the two processes 
show the same scale characteristics. With the measurements quoted above this yields $d v / d L=4-$ $4-20 \times 10^{3}$ and $f_{L} / f_{V}=9-25 \times 10^{3}$, in excellent agreement. Although somewhat simplistic, this first order treatment confirms that our laboratory data scales to natural volcanic data. Likewise, other parameters relevant to volcano physics, such as viscosity $(V)$, can also be scaled in this simple way. Using $V_{L}=10^{-2}$ poise for laboratory pore water and $V_{V}=10^{3}$ poise for basaltic lava, respectively, and length scales of $d \mathrm{~L}=50 \mathrm{~mm}$ and $\mathrm{dv}=10 \mathrm{~km}$ (common for effusive eruptions), we find that $d v / d_{L}=2 \times 10^{5}$ and $V_{V} / V_{L}=1 \times 10^{5}$; again providing excellent agreement.

We further propose that the recorded low frequency AE events acted as individual trigger sites in our sample for the longer LF tail. This hypothesis is supported by the locations of individual LF events, which are not confined to a particular site within the sample damage zone, indicating different sources within the sample. The source of the LF tremor induced by the trigger swarm is therefore likely to be related to the conduit itself (8). This can be verified using the dimensions of the laboratory setup and the simple relationship: velocity $=f \lambda$. For the known conduit length $(\lambda)$ of $125 \mathrm{~mm}$ and a compressional wave velocity ranging from $1500 \mathrm{~m} / \mathrm{s}$ (water) to $6000 \mathrm{~m} / \mathrm{s}$ (unfractured basalt), frequencies ( $f$ ) are between $12 \mathrm{kHz}$ and $48 \mathrm{kHz}$. This is in good agreement with the recorded resonances of approximately $20-50 \mathrm{kHz}$. Our laboratory data therefore support field evidence $(1,18,25,29)$, high temperature laboratory evidence on partial melts $(26)$ and model evidence $(4,8,13)$ that LF events are generated as a combined result of fluid interactions with both the damage zone and the conduit. 


\section{References and notes}

1. B. A. Chouet, Nature 380, 309 (1996).

2. H. Kumagai et al., Science 293, 687 (2001).

3. B. Voight et al., Science 283, 1138 (1999).

4. B. Chouet, Pure and Applied Geophysics 160, 739 (2003).

5. C. R. J. Kilburn, J. Volc. Geotherm. Res. 125, 271 (2003).

6. S. R. McNutt, in Monitoring and mitigation of volcanic of volcanoes, R. Scarpa, R. Tilling, Eds. (Springer \& Verlag, New York, 1996).

7. R. Scandone, K.V. Cashman, S.D. Malone, EPSL, 253, 513 (2007).

8. J. W. Neuberg et al., J. Volc. Geotherm. Res. 153, 37 (2006).

9. G. Rogers, H. Dragert, Science 300, 1942 (2007).

10. Y. Ito et al., Science 315, 503 (2007).

11. P. M. Benson et al., Geophys. Res. Lett. 34, 10.1029/2006GL028721 (2007).

12. $A E$ has its origin in mechanical engineering and high pressure hydraulics, where it is frequently employed to detect leaks from gas tanks (e.g. LPG flashing to gas). The turbulent flow generates $A E(S 1)$.

13. K. Aki, P. Richards, Quantitative Seismology (University Science Books, Sausalito, CA, ed. 2, 2002).

14. D. Patane et al., Science 299, 2061 (2003).

15. P. M. Benson, S. Vinciguerra, P. G. Meredith, and R. P. Young, paper presented the 29th Course of the International School of Geophysics Euro-Conference of Rock Physics and Geomechanics, Erice, Sicily, 28th September, 2007.

16. D. C. Collins, W. S. Pettitt, R. P. Young, Pure appl. geophys. 159, 197 (2002).

17. S. Stanchits et al., Pure appl. geophys. 163, 974 (2006).

18. S. Miller et al., Nature 427, 10.1038/nature02251 (2004).

19. D. A. Lockner et al., Nature 350, 39 (1991). 
20. G. R. Foulger et al., J. Volc. Geotherm. Res. 132, 45 (2004).

21. H. Kumagai, B.A. Chouet, M. Nakano, J. Geophys. Res. 107 10.1029/2001JB001704, (2002).

22. M. R. James et al., J. Volc. Geotherm. Res. 129, 61 (2004).

23. M.R. James et al., J. Geophys. Res. 111, 10.1029/2005JB003718 (2006).

24. B. R. Julian, J. Geophys. Res. 99, 10.1029/93JB03129 (1994).

25. H. Tuffen, D. Dingwell, Bull. Volcanol. 67, 370 (2005).

26. L. Burlini et al., Geology, 35, 183 (2007).

27. I. G. Main, Nature 357, 27 (1992).

28. C. G. Hatton, I.G. Main, P. G. Meredith, Nature 367, 160 (1994).

29. C. A. Rowe et al., Geophys. Res. Lett. 25, 2297 (1998).

30. This work was supported by a Marie-Curie International Fellowship within the $6^{\text {th }}$ European Community Framework programme (Contract MOIF-CT-2005-020167 to PMB) and a Canadian Foundation for Innovation (CFI) award to RPY. The authors thank Luca Malagnini and Jurgen Neuberg for fruitful discussions and two anonymous reviewers for comments that greatly helped to improve this manuscript. 


\section{Figure and Captions}
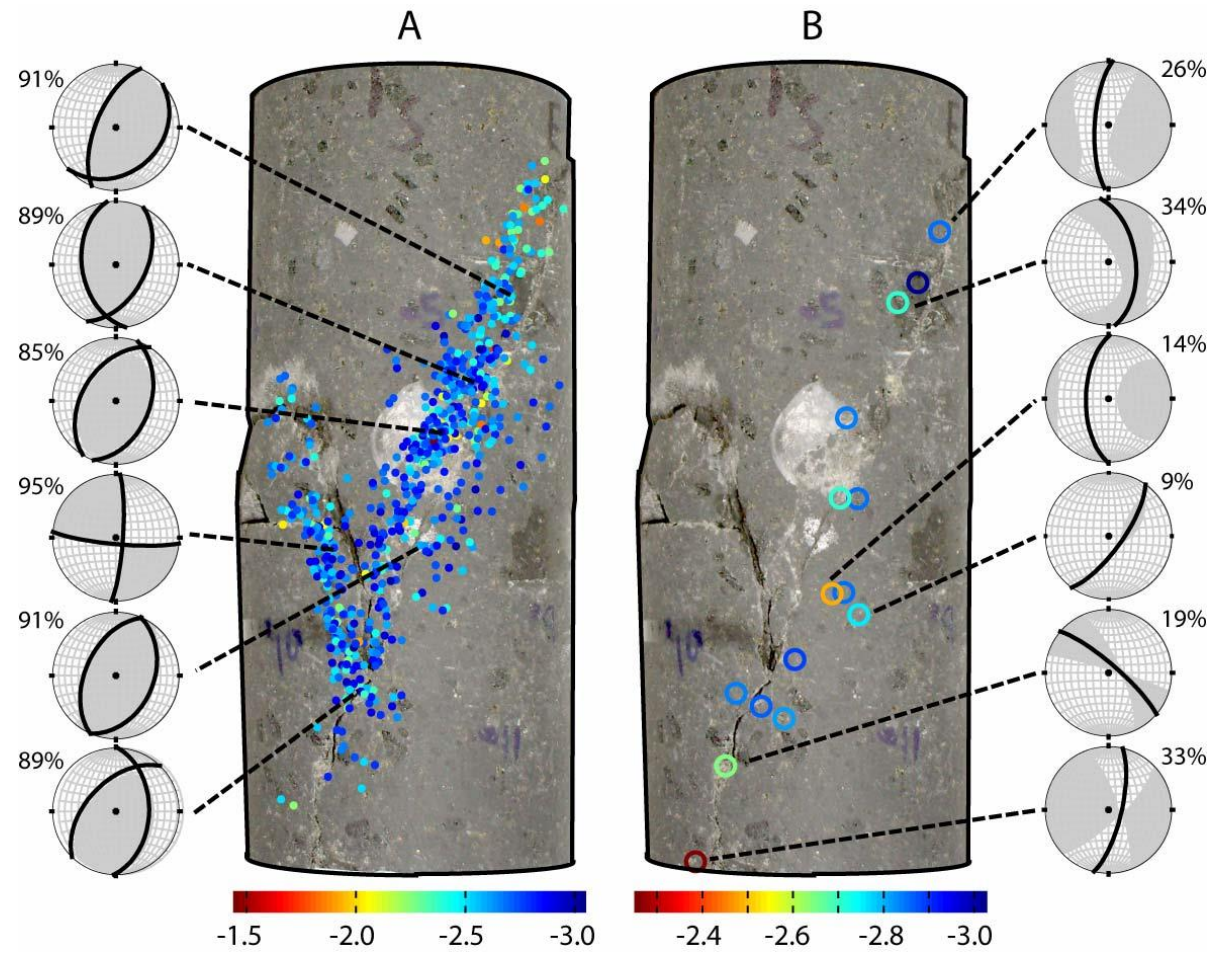

Fig. 1. Post-test sample of Etna basalt showing a single through-going fault and its conjugate. Superimposed are $A E$ locations resulting from the deformation phase of the experiment $(A$, dots), and $A E$ locations resulting from the rapid decompression of the pore fluid ( $B$, open circles). The color-bar indicates dimensionless event pseudo-magnitude, calculated using an average weighted ray-path over all receivers (19). Irrespective of the stage of experiment (deformation or decompression) events are located on or close by the fault. Source characteristics (mechanisms) associated with deformation (left) exhibit high percentage components of double couple (shear); whereas those relating to pore fluid decompression (right) show much lower double couple components. 


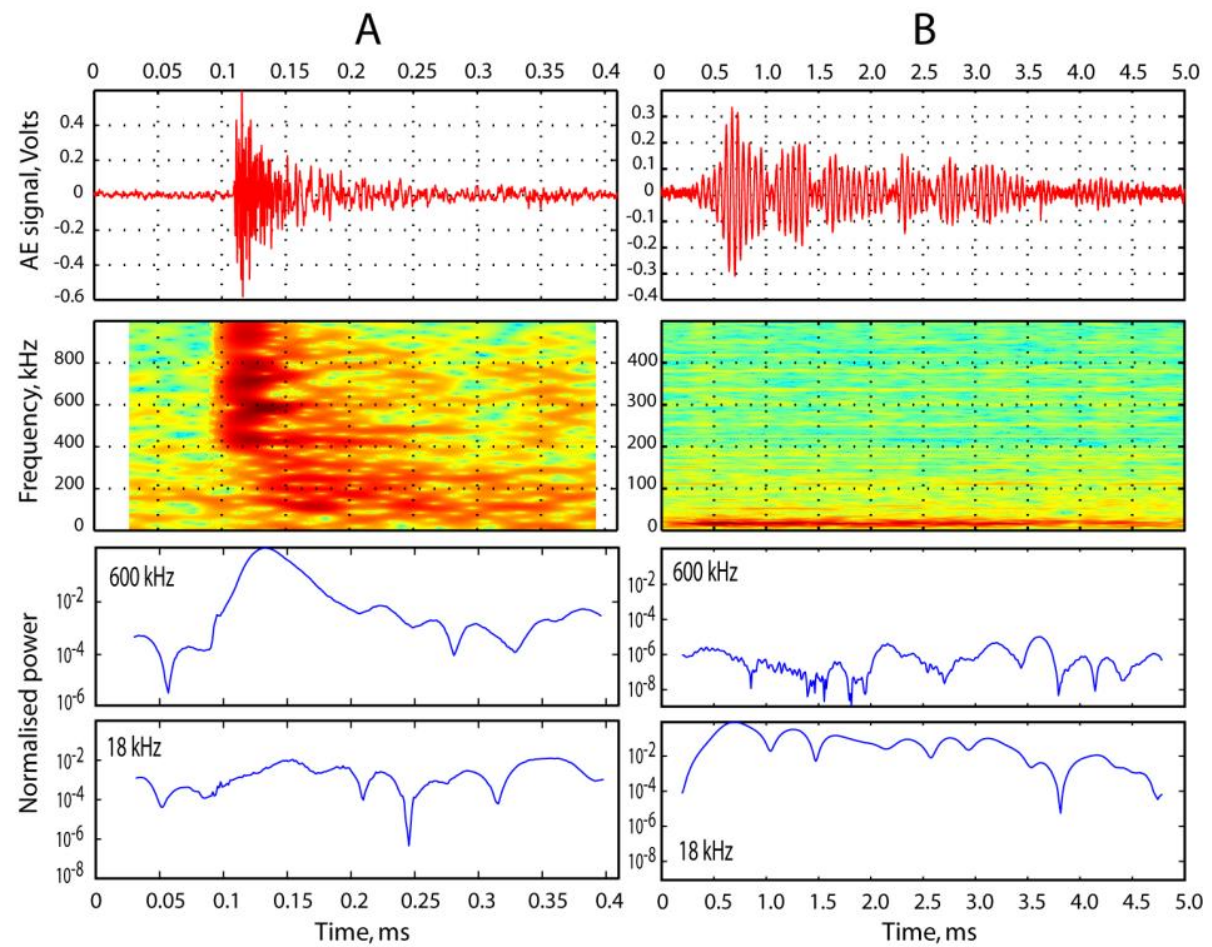

Fig. 2. Representative waveform types observed during the experiment. A: High frequency events analogous to VT (volcano tectonic) seismicity are observed during sample deformation and fracture formation. These signals have a maximum power component at $600 \mathrm{kHz}$ (and above), with less power at lower frequencies. B: 'Low Frequency' events observed during pore space decompression. These signals have a peak power component at approximately $18-20 \mathrm{kHz}$. Referring to the normalised power plots, for VT seismicity $(\mathrm{A})$ there is a rapid onset of high frequency power with a peak at $\sim 0.14 \mathrm{~ms}$ and little power seen at lower frequencies; the high frequencies die out rapidly. However, for LF frequencies there is no high frequency component to speak of, and the LF component dies very slowly, with a maximum power occurring at $\sim 0.6 \mathrm{~ms}$. 


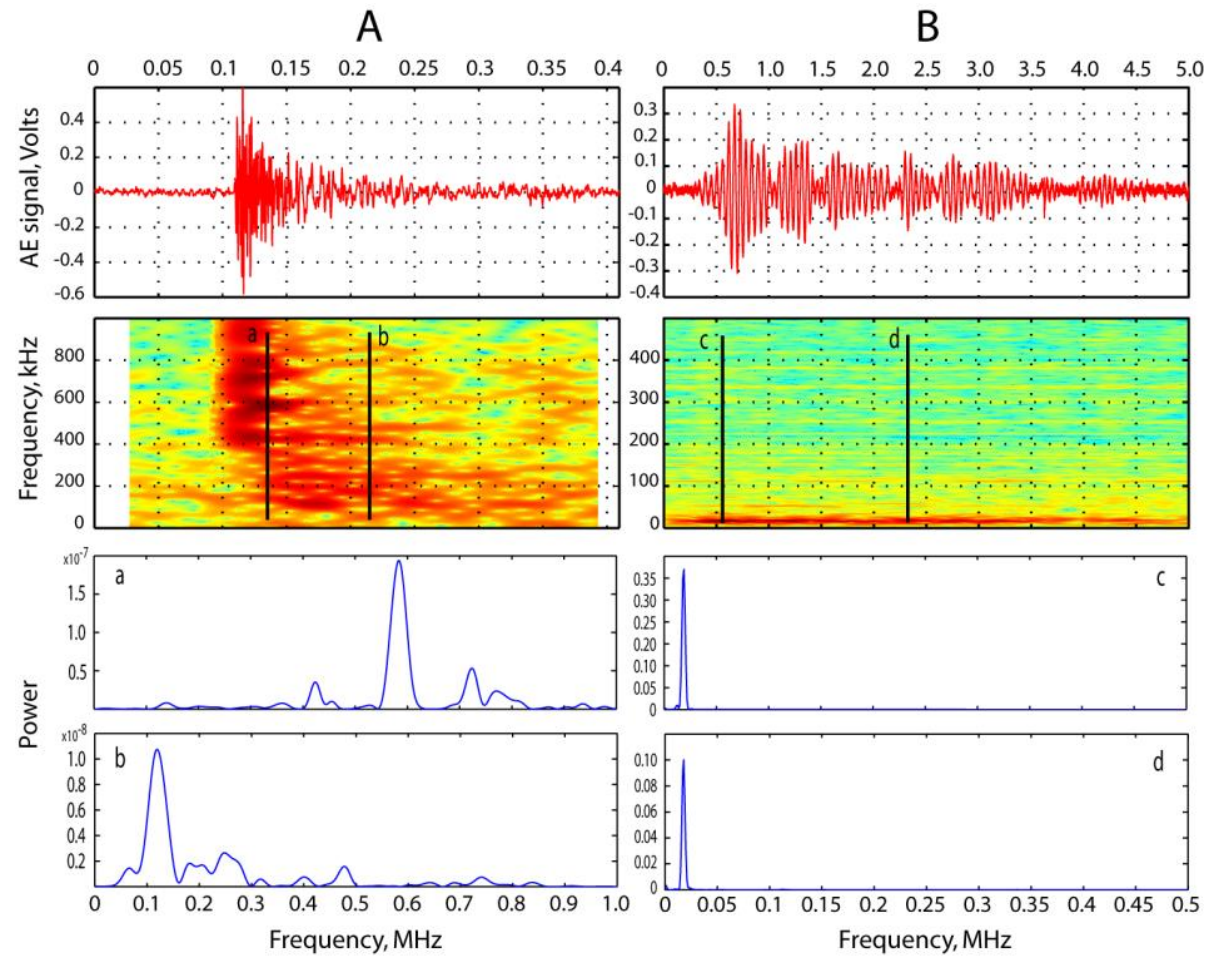

Fig. 2. ALTERNATIVE

As Stated in the response letter, we prefer the original, as this alternative version does not show as much information, as well as losing the variation of power with time. 


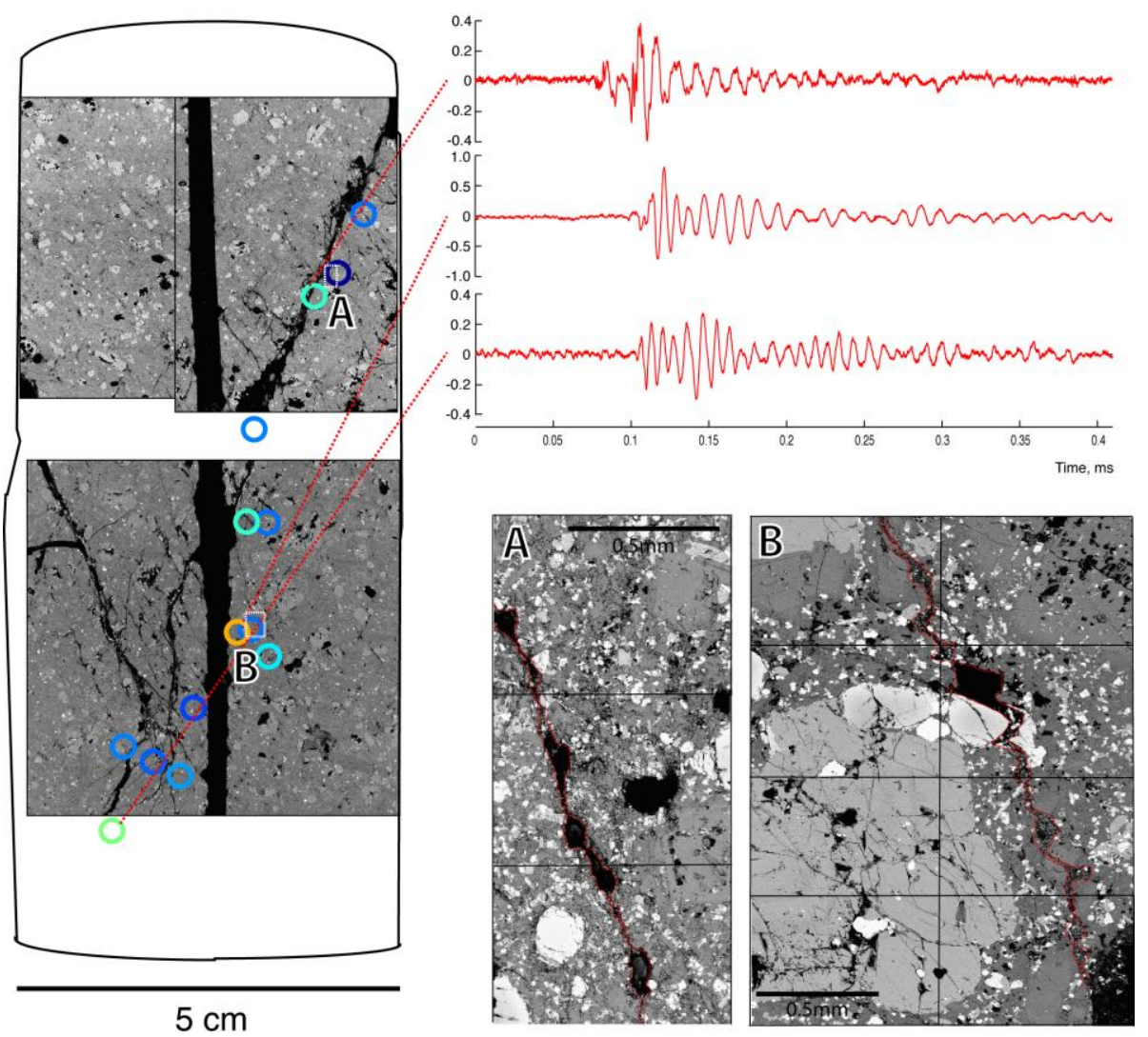

Fig. 3. SEM micrograph taken along the centre axis of the sample. (Left) AE locations from the fracture zone decompression stage (stage 2) of the experiment. Locations are sparse due to the low amplitude signals, but enough data is located to show the trend of locations within the fracture damage zone. (Top right) Some additional example waveforms, with their corresponding locations. (Bottom right) Details of the local microstructure of two areas ('A' and 'B') where clusters of LF events occurred, showing voids, undulating cracks walls and pinch-points in the damage zone. 

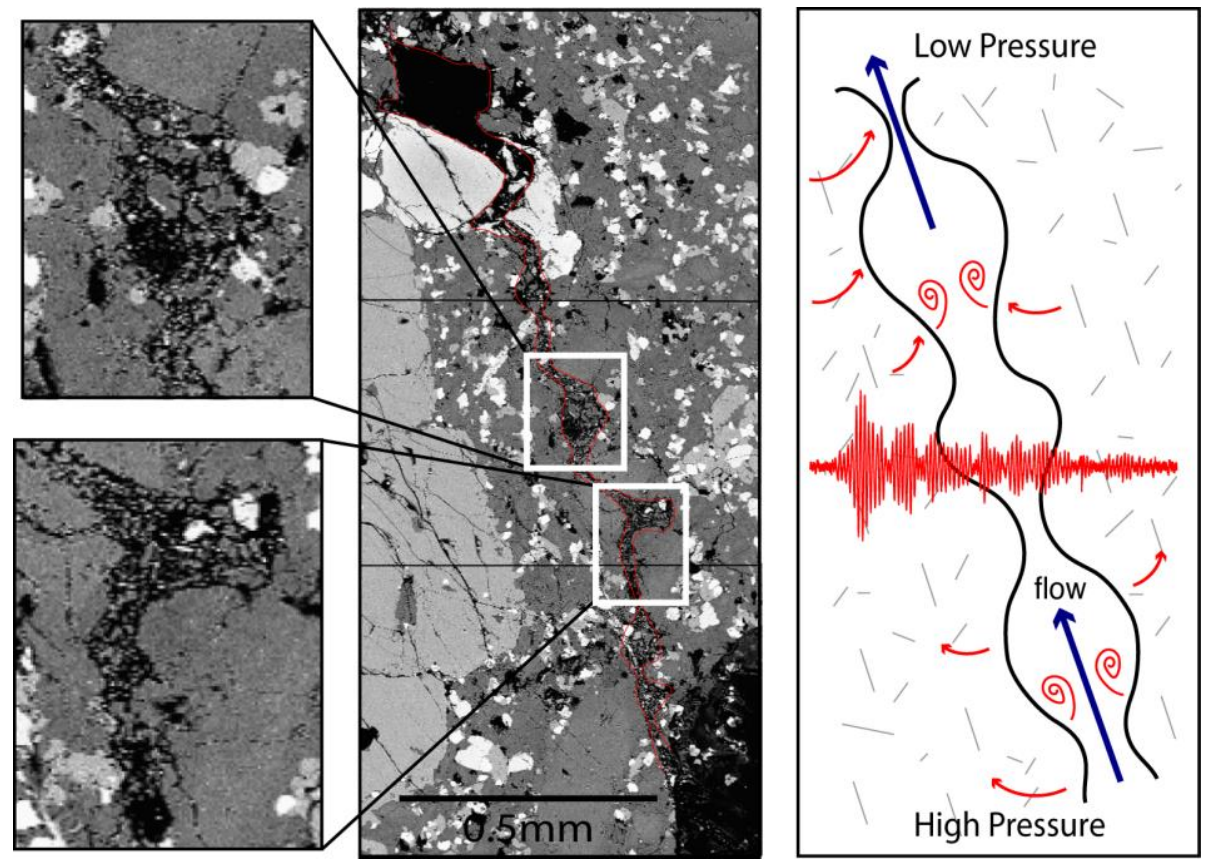

Fig. 4. (Left and Centre) Post-test laboratory micrographs showing the key features of a typical, undulating crack structure; kinks, constrictions and comminuted fill material. (Right) Conceptual model of damage zone geometry based on laboratory evidence and the tremor model of Julian (1994), with additional features taken from the aseismic plug flow model of Neuberg (2006). We also observe comminuted rock fragments within the damage zone, analogous to ash in fractures as observed in the field by Tuffen and Dingwell (2005), and hypothesized by Chouet (1996). It is likely that any non-laminar flow produced by the undulations and kinks in the damage zone is recorded as LF seismicity due to conduit/crack resonance, as these features are commonly seen at the location of the LF AE events in our experiment. 


\title{
Supporting Online Material for
}

\section{Laboratory Simulation of Volcano Seismicity}

\author{
Philip M Benson ${ }^{1,2^{\star}}$, Sergio Vinciguerra ${ }^{3}$, Philip G Meredith ${ }^{1}$ and R Paul Young ${ }^{2}$ \\ ${ }^{*}$ Corresponding author: p.benson@ucl.ac.uk
}

This file includes:

Figs. S1 to S3

References

Purpose of this supporting material

This supporting online material contains 3 figures with captions designed to aid the reader in understanding the experimental setup described in the accompanying paper. 


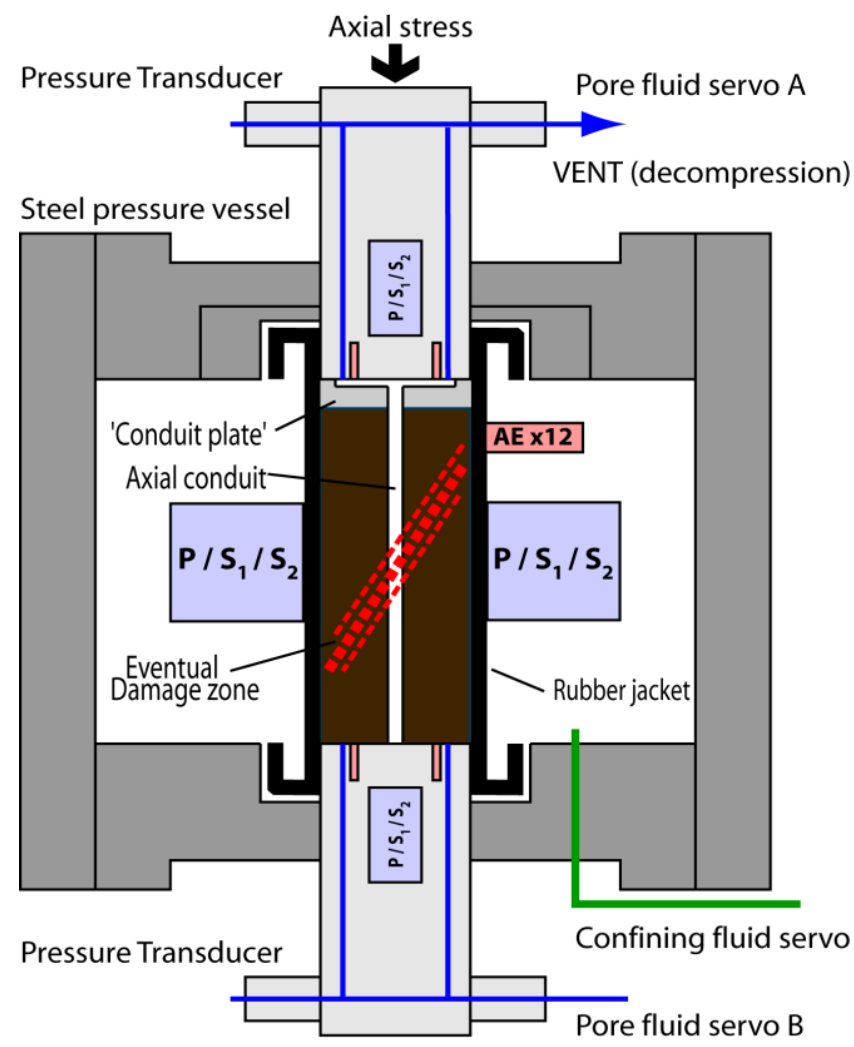

Fig. S1. Experimental setup. Detail of a sample and experimental setup, with annotation shown for the axial conduit and the fault motion due to applied stress. The axial conduit is drilled in order to provide direct access to the fault damage zone in order to stimulate decompression of the zone as rapidly as possible. Sample dimensions are $50 \mathrm{~mm}$ diameter by $125 \mathrm{~mm}$ length. A confining pressure of $60 \mathrm{MPa}$ (approximately $2 \mathrm{~km}$ depth) was used, with a pore pressure of $20 \mathrm{MPa}(0.8$ $\mathrm{km})$. An array of 16 piezoelectric sensors to detect Acoustic Emission (AE) was arrayed around the sample and embedded in the upper and lower steel platens. AE has its origin in mechanical engineering and high pressure hydraulics, where it is frequently employed to detect leaks from gas tanks (e.g. LPG flashing to gas). The turbulent flow produced generates AE (S1). In order to determine an accurate velocity model for source location inversion, a triaxial array of P-wave transducers was employed. A rubber jacket isolates the sample from the silicone oil confining medium. 


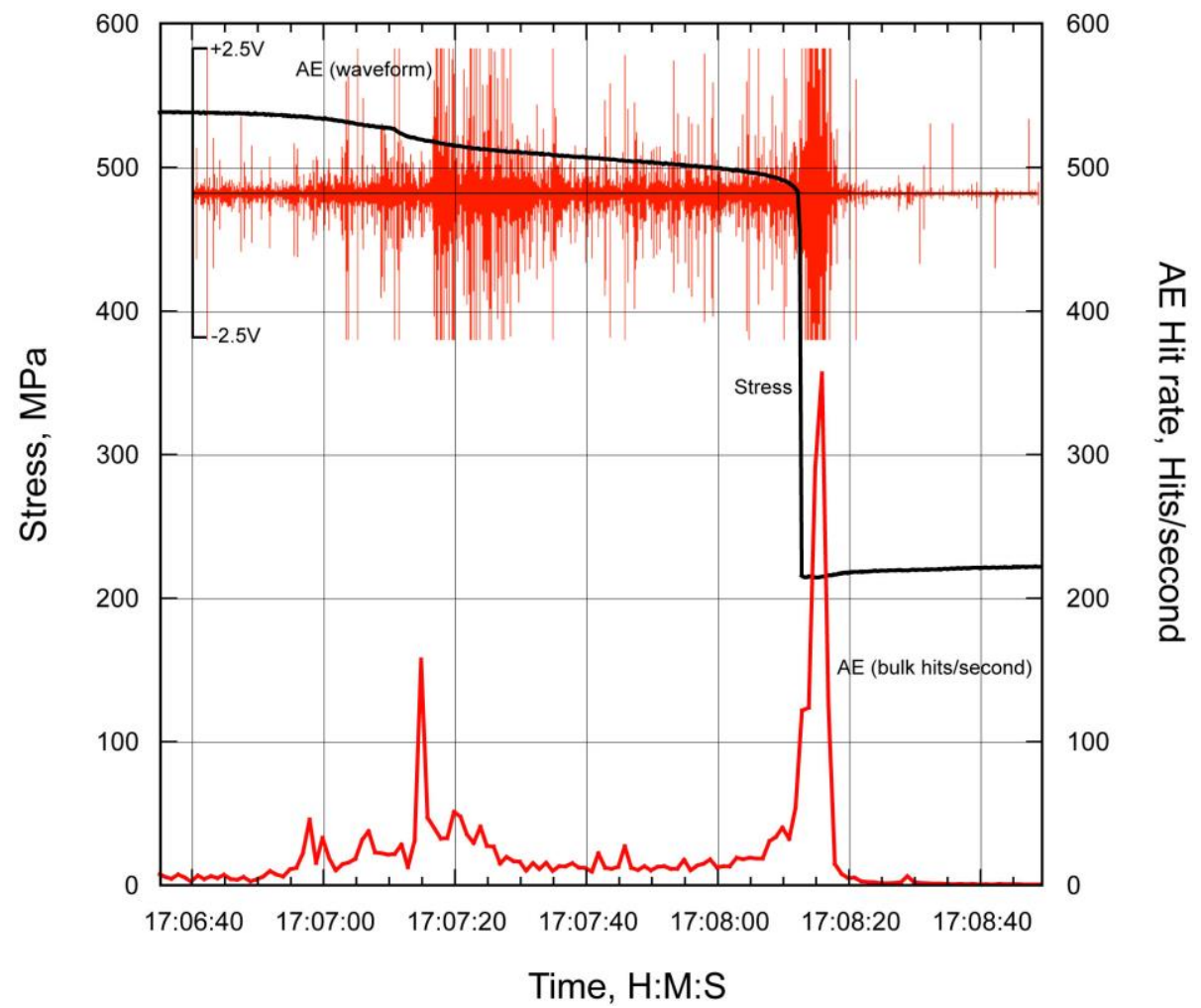

Fig. S2. Stress-time (left axis) and AE hit rate (right axis) data for stage 1 of the experiment, with AE waveform superimposed during the key $\sim 2$ minute period of fault coalescence, nucleation and failure. The sample fails at approximately $480 \mathrm{MPa}$ stress and at a strain of $2.9 \%$ (not shown). AE hit rate peaks during the two stress drops, at 17:07:15s and 17:08:15s during which the fault is forming. This continuous record is later harvested for location and relative amplitude source mechanism analysis. 


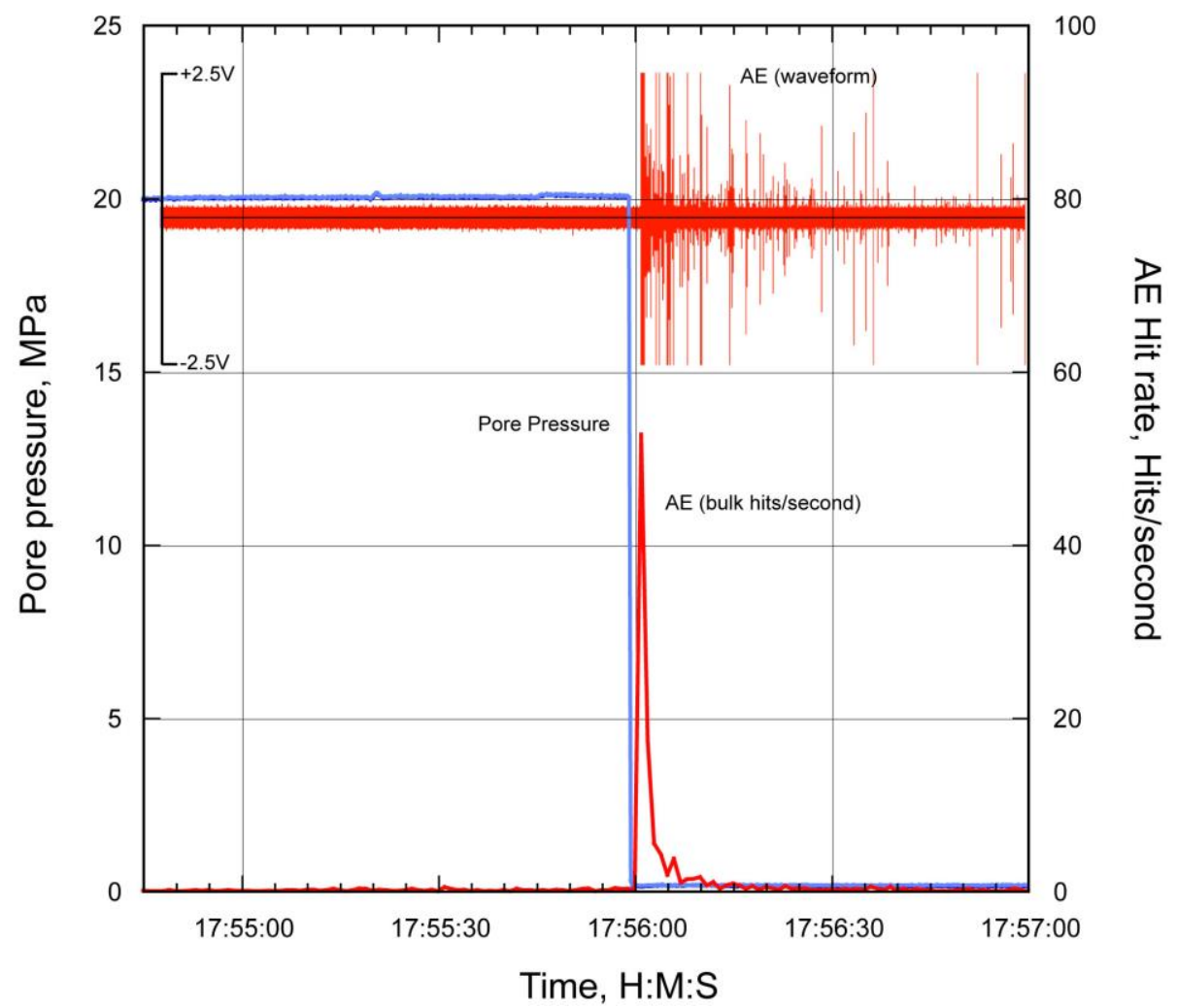

Fig. S3. Pore pressure-time (left axis) and $A E$ hit rate (right axis) data for stage 2 of the experiment, with $\mathrm{AE}$ waveform superimposed during the rapid depressurisation of the damage zone. This results in a swarm of seismicity at 17:56:00, and a peak in AE hit rate. This continuous record is later harvested for location and relative amplitude source mechanism analysis.

\section{References and Notes}

S1. M.F. Carlos, "Overview of Current and Developing ASTM Acoustic Emission Standards" (Physical Acoustics Corporation, Princeton Jct., NJ, 2004). [Unpublished or access by title] 